\title{
Eficacia y residualidad de Fluopyram + Tebuconazol en el control del oídio de uva de mesa en Piura
}

\section{Efficacy and residuality of Fluopyram +} Tebuconazole in the control of powdery mildew of table grapes in Piura

iD 'Javier Javier-Alva

Universidad Nacional de Piura. Piura, Perú.

\section{Resumen}

El oídio de la vid, enfermedad endémica limitante para el rendimiento y calidad de la uva de mesa en Piura. Esta investigación tuvo como objetivo evaluar la eficacia y residualidad de fungicidas sobre el oídio en hojas de plantas de vid Red Globe. En laboratorio, los fungicidas se aplicaron por aspersión en hojas con alta presión de enfermedad y se evaluaron las conidias viables durante 15 días. En campo, los fungicidas se aplicaron al follaje 19 días después de la cianamida y se hicieron evaluaciones hasta los 20 días. De los resultados porcentuales se obtuvo el ABCPE y, el análisis de varianza y los promedios se separaron de acuerdo con la prueba de Duncan $(p<0,05)$ utilizando el software estadístico SPSS 20. Los resultados en laboratorio demostraron que Fluopyram + Tebuconazol $(\mathrm{F}+\mathrm{T})$ al 0,10\% aplicado como curativo ejerció una significativa eficacia de control, 75,77\% $(\mathrm{p}=0,01)$ y un periodo de residualidad de ocho días. $\mathrm{F}+\mathrm{T} 0,6 \mathrm{~L} /$ ha demostró alta eficacia de control en campo como preventivo, $79,59 \%, 82,51 \%$ y $88,87 \%$ superior a Triadimenol medido por los porcentajes de incidencia, severidad e inóculo viable, respectivamente $(p=0,01)$. $F+T$ tuvo el periodo de residualidad más alto en campo, 11 días estudiando la incidencia y severidad, y 8 días estudiando el inoculo viable. $\mathrm{F}+\mathrm{T}$ es una nueva herramienta en el programa de manejo integrado del oídio de la vid.

Palabras clave: Conidias viables, incidencia, severidad, inóculo viable, eficacia, residualidad.

\section{Abstract}

The vineyard mildew, limiting endemic disease for the table grapes crop yield and quality in Piura. This research aimed to evaluate the fungicides efficacy and residuality on powdery mildew in Red Globe vine plants leaves. In the labora-

Recibido: Marzo 2020

Aceptado: Junio 2020 tory, fungicides were applied by spraying on leaves with high disease pressure and viable conidia were evaluated for 15 days. In the field, the fungicides were applied to the foliage 19 days after the cyanamide and evaluations were made up to 20 days. ABCPE was obtained from the percentage results and the analysis of variance and averages were separated according to the Duncan test $(p<0.05)$ using the statistical software SPSS 20. The laboratory results showed that $0.10 \%$ 
Fluopyram + Tebuconazole ( $\mathrm{F}+\mathrm{T}$ ) applied as a curative exerted a significant control efficacy, $75.77 \%(p=0.01)$ and eight days' residual period. $F+T 0.6 \mathrm{~L} /$ ha demonstrated high control effectiveness in the field as preventive, $79.59 \%, 82.51 \%$ and $88.87 \%$ higher than Triadimenol measured by the percentages of incidence, severity and viable inoculum, respectively $(p=0.01)$. $F+T$ had the highest residual period in the field, 11 days studying the incidence and severity, and 8 days studying the viable inoculum. $\mathrm{F}+\mathrm{T}$ is a new tool in the integrated management program of the vineyard.

Keywords: Viable conidia, incidence, severity, viable inoculum, efficacy, residuality

\section{INTRODUCCIÓN}

El oídio causado por Oidium tuckeri Berk., anamorfo de Erysiphe necator Schw. es una enfermedad endémica limitante para el cultivo de vid (Vitis vinifera) para uva de mesa en Piura - Perú caracterizada por un clima seco. Su importancia se debe, principalmente a que detiene el crecimiento de la piel de los granos de uva, lo cual provoca agrietamientos y roturas que permiten la entrada de hongos y bacterias que causan pudriciones. O. tuckeri es un parásito obligado biotrófico, con alta especificidad en la vid, afectando tejidos tiernos como hojas, tallos, frutos e inflorescencias. Bajo condiciones de Piura, el oídio sobrevive como micelio con apresorios lobados sobre la superficie adaxial de los profilos en los interiores de yemas verdes en los nudos 3 y 5 , de las cuales emerge cuando los brotes bandera empiezan a crecer (Pearson and Gärtel, 1985). A temperaturas entre $18-30^{\circ} \mathrm{C}$ la infección secundaria ocurre inmediatamente, produciendo conidias 7-10 días después y, si las condiciones climáticas son favorables, la producción de nuevas conidias es de 5 días (Gubler et al., 1999). De acuerdo a esto, el manejo de la enfermedad en Piura, se basa en aplicaciones de fungicidas desde la emergencia del brote bandera, alternando fungicidas curativos y preventivos para mantener bajo inóculo cuando se llega al periodo más crítico que es entre inicios de la floración y bayas pequeñas (Campbell et al., 2007). La aplicación de fungicidas continuará siendo una herramienta prominente para el manejo del oídio (Gadoury et al, 2012) Sin embargo, la escasa eficacia y residualidad de los fungicidas de acción específica usados con frecuencia para el control de esta enfermedad en Piura que da lugar a la emergencia de resistencia como la reportada para Metrafenona en Italia (Kunova et al., 2015) y las aplicaciones tardías constituyen un serio problema que es necesario estudiar para establecer nuevas estrategias de manejo. En esta investigación, en busca de reducir el número de aplicaciones de fungicidas, se planteó estudiar la eficacia y residualidad del fungicida Luna Experience sobre la viabilidad de conidias bajo condiciones de alto potencial de inóculo inicial en laboratorio y, evaluar en campo su eficacia y residualidad basada sobre la incidencia, severidad e inóculo viable del oídio comparados con otros fungicidas comerciales del mercado.

\section{MATERIALES Y METODOS Fase de laboratorio: Estudio de conidias viables}

Se desarrolló en el laboratorio de Fitopatología de la Universidad Nacional de Piura.

Inoculación de conidias. En hojas jóvenes de plantas de vid variedad Red Globe de cuatro meses previamente lavadas cuidadosamente con aspersor manual para remover las partículas de polvo. El inóculo se obtuvo a partir de hojas con signos de oidio procedentes del campo, deslizando suavemente un hisopo de algodón estéril sobre el área de esporulación. La inoculación también se hizo deslizando suavemente el hisopo sobre la cara inferior de 
hojas jóvenes sanas. Las plantas se mantuvieron durante un mes en un lugar con periodos alternados de sombra y Sol durante el día hasta la aparición de síntomas y signos.

Fungicidas utilizados. Luna Experience (Fluopyram $200 \mathrm{~g} / \mathrm{L}$ PC + Tebuconazol $200 \mathrm{~g} / \mathrm{L}$ PC al 0,1\% de producto comercial), Prosper (Spiroxamine $500 \mathrm{~g} / \mathrm{L} \mathrm{PC}$ ) al 0,06\% de producto comercial (PC) + Serenade ASO 1,34 SC (BaciIlus subtilis cepa QST 713; 1,34\%) al 0,5\% de PC, Vydan (Triadimenol $250 \mathrm{~g} / \mathrm{L}$ ) al 0,5\% de PC y el Testigo (agua) Aplicación de fungicidas. Una sola vez al envés de las hojas dirigida a la zona de esporulación utilizando un micro aspersor manual.

Evaluación de conidias viables. A 0, 1, 2, 3, 4, 5, $6,7,8,9,10,11,12,13,14$ y 15 días después de la aplicación de los tratamientos. La captura de conidias se hizo utilizando cinta adhesiva transparente, colocando la parte adhesiva sobre la zona de esporulación en el envés de las hojas, presionando suavemente y luego despegando. La parte adhesiva conteniendo el inóculo se colocó sobre una lámina portaobjetos, sobre la cual previamente había una gota de agua destilada estéril extendida sobre la lámina. Las observaciones microscópicas se hicieron a mediano aumento (20X) en cuatro campos diferentes, contando en cada campo el número total de conidias (NTC) y el número de conidias viables (NCV). El porcentaje de conidias viables en cada hoja y en cada momento de observación se obtuvo mediante la fórmula:

$$
\% \mathrm{CV}=(\mathrm{NCV} / \mathrm{NTC}) * 100
$$

Se consideró viable aquella turgente, hialina y sin daños observables y, no viable aquella con deformaciones producto de la plasmólisis por efecto de fungicidas (Fig. 1).
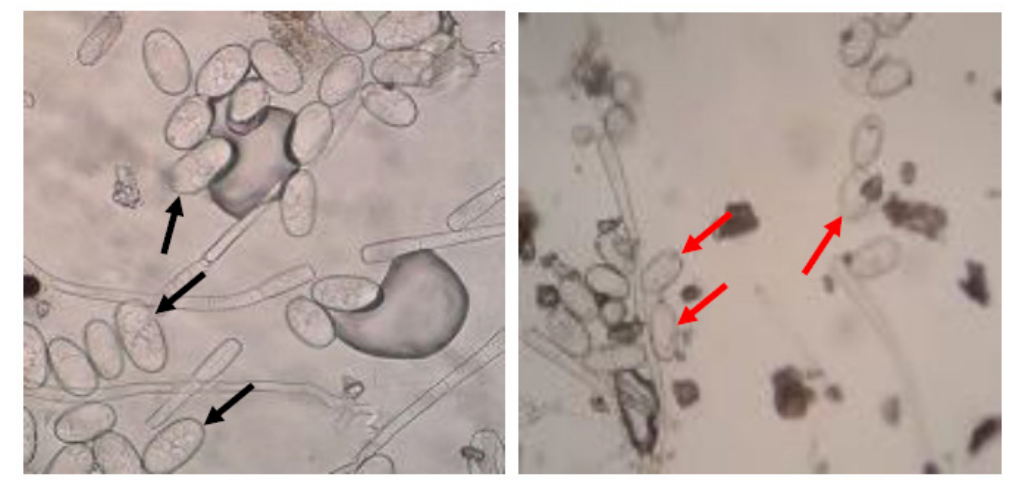

Figura 1. Conidias viables turgentes señaladas con flechas negras hacia la izquierda y conidias no viables señaladas con flechas rojas hacia la derecha (20X).

Diseño y análisis estadístico. Los tratamientos se distribuyeron en un Diseño Completamente al Azar con empleando como unidad experimental cuatro hojas por planta. El efecto de cada fungicida se evaluó diariamente por medio del porcentaje de conidias viables. Se calculó el Área Bajo la curva Para el Progreso de la enfermedad (ABCPE) y ABCPE estandarizada (ABCPEE) como un resumen cuantitativo del porcentaje de conidias viables para la comparación de los fungicidas con el tiempo en días (Campbell y Madden, 1989). Los resultados se sometieron al análisis de varianza y los promedios se separaron de acuerdo con la prueba de Duncan $(p<0,05)$ utilizando el software estadístico SPSS 20.

Determinación de la eficacia. Fue calculada en base a la fórmula de Abbott (1925):

$\%$ de eficacia $=\left[\left(I_{c} I_{t}\right) / I_{c}\right] \times 100$, donde $I_{c}$ es la incidencia de conidias viables del control, l es la incidencia de conidias viables en el tratamiento. 
Determinación de la residualidad. Para la determinación del periodo de residualidad, se graficó el porcentaje de conidias viables vs días de observación.

\section{Fase de campo}

Lugar de ejecución. Empresa agrícola ECOSAC, caserío Chapairá-Medio Piura, cultivo de vid Red Globe/Salt Creek de 4 años, 1250 plantas/ha, suelo franco arenoso.

Determinación del índice de Riesgo del Oídio (IRO). Registrando la temperatura y humedad relativa durante los 20 días de estudio de la estación meteorológica de Bayer Grape- net instalada en Sociedad Agrícola Rapel de acuerdo a Gubler et al. (1999).

Labores previas. Poda para producción el 17 de junio y aplicación de Cianamida el 18 de junio. El 1 de julio se aplicó Microthiol (azufre) 1,5 kg/ha y el deshoje el 22 de julio. Se eligieron al azar tres lotes de plantas y se marcaron al azar 12 plantas por lote, seis hojas por planta entre los nudos 3 y 4 de brotes de 18-20 días de edad.

Aplicación de fungicidas. Al follaje con máquina pulverizadora de la empresa con un gasto de agua de 600 L/ha (Tabla 1).

Tabla 1. Cronograma de aplicaciones de fungicidas para el control del oídio en hojas de vid en campo

\begin{tabular}{|c|c|c|c|c|c|c|}
\hline \multirow[b]{2}{*}{ Trat. } & \multicolumn{6}{|c|}{ Días (Fecha) } \\
\hline & Primera: $0(07 / 07)$ & $\begin{array}{l}\text { Dosis } \\
\text { (L/ha) }\end{array}$ & Segunda: $12(19 / 07)$ & $\begin{array}{l}\text { Dosis } \\
(\mathrm{L} / \mathrm{ha})\end{array}$ & Tercera: $16(23 / 07)$ & $\begin{array}{l}\text { Dosis } \\
(\mathrm{L} / \mathrm{ha})\end{array}$ \\
\hline 1 & $\begin{array}{l}\text { Fluopyram + } \\
\text { Tebuconazol }^{1}\end{array}$ & 0,6 & Spiroxamine & 0,36 & $\begin{array}{c}\text { Tebuconazol + } \\
\text { Trifloxystrobin }^{6}\end{array}$ & 0,350 \\
\hline 2 & $\begin{array}{c}\text { Spiroxamine }^{2}+ \\
\text { B. subtilis }\end{array}$ & $\begin{array}{l}0,36 \\
3,0\end{array}$ & Metrafenona ${ }^{5}$ & 0,3 & $\begin{array}{l}\text { Tebuconazol + } \\
\text { Trifloxystrobin }\end{array}$ & 0,350 \\
\hline 3 & Triadimenol $^{4}$ & 0,3 & Spiroxamine & 0,36 & $\begin{array}{l}\text { Tebuconazol + } \\
\text { Trifloxystrobin }\end{array}$ & 0,350 \\
\hline
\end{tabular}

Diseño y análisis estadístico. Los tratamientos se distribuyeron en un Diseño en Bloques Completamente al Azar con tres repeticiones y como unidad experimental seis hojas por planta. Las evaluaciones se hicieron a $0,1,4$, $6,8,11,13,15,18$ y 20 días. El porcentaje de incidencia (PI) se calculó con la fórmula I (\%) $=100 \times\left(n^{\circ}\right.$ de hojas infectadas $/ n^{\circ}$ de hojas evaluadas). La severidad de la enfermedad se evaluó con una escala de 0 a 5 grados, teniendo en cuenta el área total del haz de la hoja afectada: 0: hoja sana y 1 a 5 hojas afectadas con oídio: 1: $>0$ hasta 5\%, 2: $>5$ hasta 12,5\%, 3: $>12,5$ hasta 25\%, 4: $>25$ hasta el 50\%, 5:>50\% (Fig. 2). El porcentaje severidad (PS) se calculó mediante la fórmula de Townsend y Heuberger (1943): PS (\%) $=(\Sigma$ n.v. $/ 6 \mathrm{~N}) \times 100$, donde $n$ : número de hojas en cada grado, v: grado de severidad o infección, N: número de hojas totales (Fig. 2). El inóculo viable se evaluó con la misma escala de 0 a 5 grados, de acuerdo a la proporción de inóculo viable estimado en la zona de esporulación, utilizando lupas de 15 aumentos para visualizar a detalle el signo del patógeno en el haz o envés, permitiendo distinguir si tenía un color blanquecino que indicaba viabilidad o marrón que indicaba no viabilidad, sin importar el tamaño en la zona de esporulación (Fig. 3). El porcentaje de inóculo viable (PIV) también se calculó mediante la 
fórmula de Townsend y Heuberger (1943). Con los valores del PI, PS y PIV se calculó el ABCPE y el $A B C P E$ como un resumen cuantitativo para la comparación de los fungicidas con el tiempo en días (Campbell y Madden, 1989) y luego se transformaron a $\sqrt{ } \mathrm{x}+1$ antes de realizar los análisis respectivos. Los resultados se sometieron al ANVA y los promedios se separaron de acuerdo con la prueba de Duncan $(p<0,05)$ utilizando el software estadístico SPSS 20.

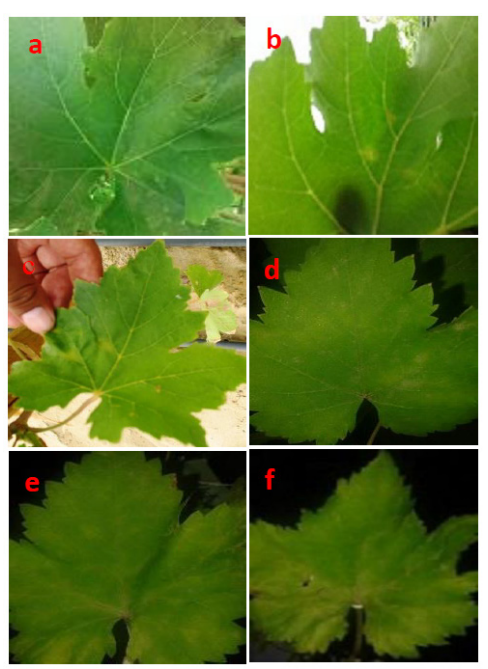

Figura 2. Escala de 0 hasta 5 grados para la evaluación de la severidad teniendo del oídio en hojas de vid, teniendo en cuenta el porcentaje del área total de las hojas afectadas con manchas amarillentas en el haz: 0: hoja sana y 1 a 5 hojas afectadas con oídio: 1: $>0$ hasta 5\%, 2: $>5$ hasta 12,5\%, 3: $>12,5$ hasta 25\%, 4: >25 hasta el 50\%, 5:>50\%.

\section{RESULTADOS}

Porcentaje de conidias viables (PCV) en laboratorio.

La mayor eficacia de control la presentaron los fungicidas sistémicos Fluopyram + Tebuconazol $(F+T)$ y Spiroxamine + B. subtilis ( $+B S)$, que demostraron una acción curativa significativa sobre las conidias viables del oídio durante los 15 días de evaluación, con valores ABCPE y ABCPE estandarizada (ABCPEE) del porcentaje de conidias viables significativamente menores $(p=0,01)$. F+T demostró una eficacia de 75,77\% superior al Testigo, 69,93\%
Determinación de la eficacia. Utilizando la fórmula de Abbott (1925) igual que para la fase de laboratorio; pero, en este caso se consideró como testigo el Triadimenol.

Determinación de la residualidad. En días, cuando en los gráficos elaborados, los promedios de los porcentajes alcanzaron los umbrales económicos de daño (UED) establecidos arbitrariamente: PI (4\%), PS (1\%) y PIV (1\%),

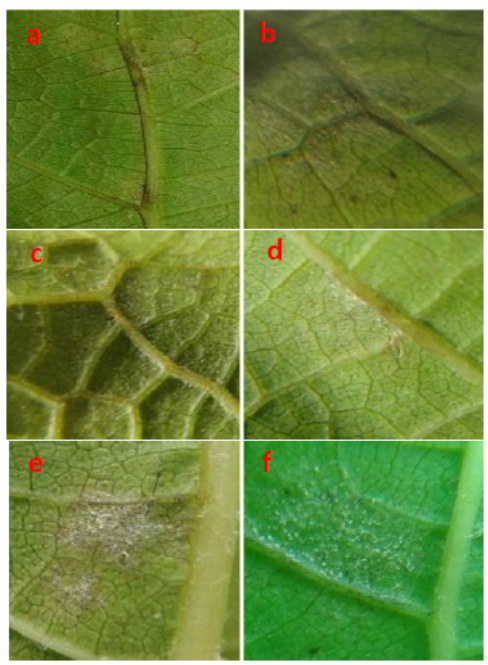

Figura 3. Escala de 0 hasta 5 grados para evaluar el inóculo viable del oídio basada en observaciones visuales con lupas de 15 aumentos para estimar la proporción de las zonas blanquecinas de esporulación. a) 0: sin signo de esporulación, b) 1:>0 hasta 5\%, c) 2: $>5$ hasta 12,5\%, d) 3: > 12,5 hasta 25\%, e) 4: $>25$ hasta $50 \%$ y f) 5: $>50 \%$.

superior a S+BS y 76,49 superior a Triadimenol. S+BS tuvo una eficacia de 19,44\% superior al Testigo y 21,82\% superior a Triadimenol (Tabla 2, Gráfico 4). El efecto curativo de F+T que se inició con un promedio de $84,14 \%$ de conidias viables al momento de la aplicación, se observó ligeramente entre el primer y segundo día y fue bien marcado a partir del tercer día. Su residualidad tuvo un periodo bien marcado de ocho días con niveles de 4 y $0 \%$ de conidias viables y se mantuvo hasta los 15 días. 


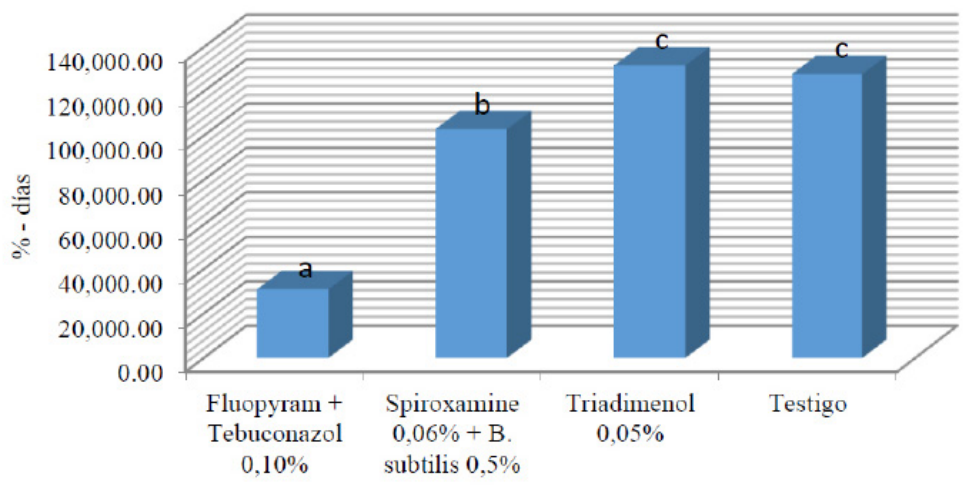

Figura 4. $A B C P E$ conidias viables en laboratorio

El efecto curativo de S+BS que al inicio tuvo un PCV de $77,07 \%$, se observó entre el primer y segundo día después de su aplicación, siendo más marcado a partir del tercer día con un PCV de 54,51\%. Si bien el PCV llegó a su nivel más bajo el séptimo día con
$34,85 \%$, su real residualidad fue de tres días con $54,51 \%$, ya que el cuarto y quinto día subió a 65 y 70\%, respectivamente. Triadimenol y el Testigo no demostraron efecto curativo durante todo el periodo de estudio (Figura 5).

Tabla 2. Prueba de Duncan para comparación de medias $(a=0.05)$ del ABCPE y ABCPE estandarizada de conidias viables

\begin{tabular}{lccccc}
\hline \multicolumn{1}{|l}{ Ingrediente activo } & $\begin{array}{c}\text { Concentración } \\
\text { P.C. (\%) }\end{array}$ & $\begin{array}{c}\text { ABCPE } \\
(\%-d i ́ a s)\end{array}$ & $\begin{array}{c}\text { ABCPEE } \\
(\%-d i ́ a)\end{array}$ & $\begin{array}{c}\text { \% respecto } \\
\text { al Testigo }\end{array}$ & \% de eficacia \\
\hline $\begin{array}{l}\text { Fluopyram } \\
\text { Tebuconazol }\end{array}$ & 0,10 & 30978,000 & 2065,20 & 24,23 & $75,77 \mathrm{a}$ \\
$\begin{array}{l}\text { Spiroxamine }+ \text { B. } \\
\text { subtilis }\end{array}$ & $0,06+0,50$ & 103011,7500 & 6867,45 & 80,56 & $19,44 \mathrm{~b}$ \\
$\begin{array}{l}\text { Testigo (Agua) } \\
\text { Triadimenol }\end{array}$ & - & 127871,2500 & 8524,73 & 100,00 & $0,00 \mathrm{c}$ \\
\hline
\end{tabular}

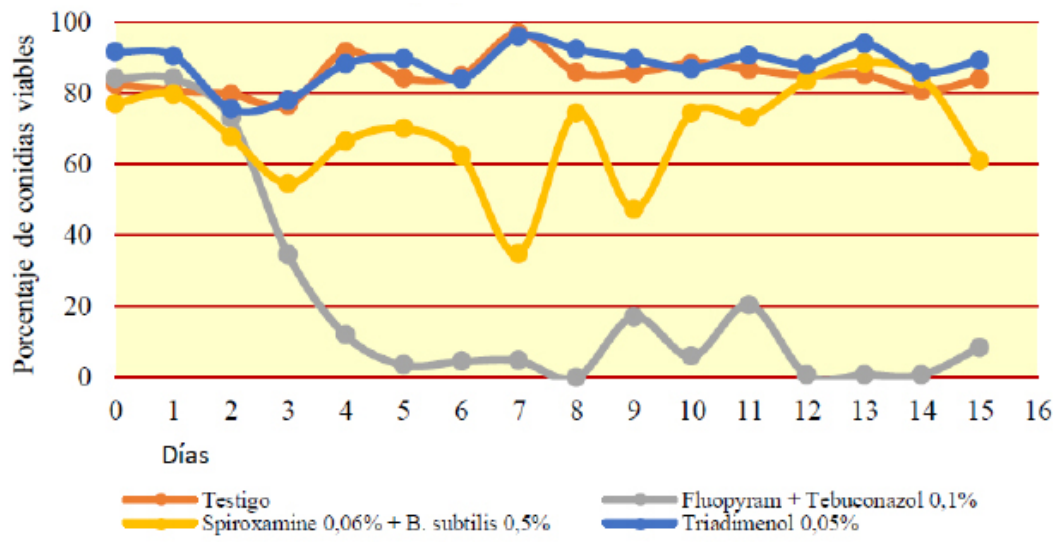

Figura 5. Porcentajes promedio de conidias viables del oídio 


\section{Fase de campo}

Índice de riesgo del oídio de la vid (IRO). EI rango de temperatura diaria durante los 16 días que se desarrolló el experimento varió desde 20,6 hasta $23,2^{\circ} \mathrm{C}$, promedio $22,23^{\circ} \mathrm{C}$. La HR varió desde 61,9 hasta $74,5 \%, 69,10 \%$. Las horas diarias que se presentaron estas condiciones de temperatura variaron desde 9 hasta 16, promedio de 12,21 h (Tabla 3). De acuerdo al IRO en vid de la Universidad de California (Gubler et al., 1999), se requieren tres días consecutivos con al menos 6 horas diarias entre $21-30{ }^{\circ} \mathrm{C}$ para activar el índice en $100 \%$, se observa que, bajo las condiciones de Piura, el IRO, se mantiene al $100 \%$ todos los días en el mes de julio cuando se dan las floraciones de la poda de junio, por lo que el patógeno se está reproduciendo cada cinco días (Figura 7).

Tabla 3. Índice de riesgo del oídio de la vid, número de horas diarias de temperaturas entre 21 y $32^{\circ} \mathrm{C}$, temperatura promedio y humedad relativa promedio (\%)

\begin{tabular}{ccccc}
\hline Fecha & $\begin{array}{c}\text { Indice de Riesgo } \\
\text { oidio }(\%)\end{array}$ & $\begin{array}{c}\text { Horas } \\
\left(21^{\circ} \mathrm{C}>=\mathrm{T}<=32^{\circ} \mathrm{C}\right)\end{array}$ & $\begin{array}{c}\text { Temperatura } \\
\text { promedio }\left({ }^{\circ} \mathrm{C}\right)\end{array}$ & $\begin{array}{c}\text { HR promedio } \\
(\%)\end{array}$ \\
\hline $01 / 07$ & 100 & 12 & 21,8 & 69,3 \\
$02 / 07$ & 100 & 12 & 22,1 & 70,2 \\
$03 / 07$ & 100 & 13 & 22,6 & 68,2 \\
$04 / 07$ & 100 & 09 & 20,6 & 74,5 \\
$05 / 07$ & 100 & 11 & 21,2 & 71,6 \\
$06 / 07$ & 100 & 11 & 21,6 & 68,5 \\
$07 / 07$ & 100 & 11 & 23,2 & 61,9 \\
$08 / 07$ & 100 & 12 & 22,2 & 70,0 \\
$09 / 07$ & 100 & 12 & 21,7 & 72,1 \\
$10 / 07$ & 100 & 12 & 22,0 & 68,6 \\
$11 / 07$ & 100 & 13 & 23,0 & 66,3 \\
$12 / 07$ & 100 & 13 & 22,2 & 70,0 \\
$13 / 07$ & 100 & 14 & 22,7 & 68,5 \\
$14 / 07$ & 100 & 16 & 23,1 & 67,4 \\
$15 / 07$ & 100 & 12 & 22,8 & 68,5 \\
$16 / 07$ & 100 & 14 & 22,4 & 70,0 \\
\hline Promedio & 100 & 12.31 & 22,23 & 69,10 \\
\hline
\end{tabular}

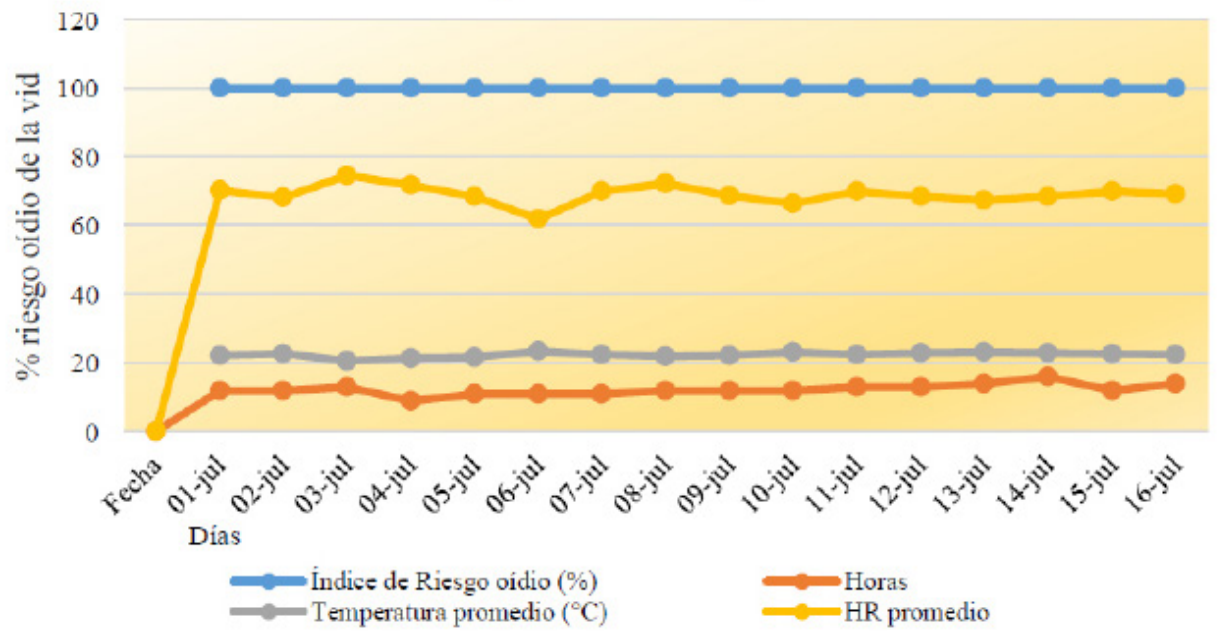

Figura 6. Índice de riesgo del oídio en hojas de vid Red Globe 
Porcentaje de incidencia del oídio. F+T demostró la más alta eficacia de control preventivo del PI del oídio en campo, con valores ABCPE y del ABCPEE significativamente menores $(p=0,01)$, $75,59 \%$ superior a Triadimenol y $64,66 \%$ superior a S+BS. La eficacia de S+BS fue 42,26\% superior a Triadimenol (Tabla 4 y Figura 7a). La residualidad de $\mathrm{F}+\mathrm{T}$ aplicado como preventivo fue de 11 días, la más alta cuando el PI llegó al UED arbitrario de $4 \%$. El periodo de residualidad del S+BS fue de 8 días y el de Triadimenol 5 días. Los niveles del porcentaje de incidencia del oídio en $\mathrm{F}+\mathrm{T}$ después del onceavo día, fueron menores hasta el final del estudio (Gráfico 4b).

Porcentaje de severidad del oídio. Eficacia más alta de control aplicada como preventivo fue demostrada por $F+T$, con valores ABCPE y ABPEE significativamente menores $(p=0,01)$, $82,51 \%$ superior al Triadimenol y $61,59 \%$ superior a S+BS. La eficacia de S+BS fue $54,47 \%$ superior a Triadimenol (Tabla 4 y Gráfico 4c). $\mathrm{F}+\mathrm{T}$ también tuvo el mayor periodo de residualidad de 11 días, estimado al alcanzar el UED arbitrario de 1\%. El periodo de residualidad de S+BS fue de 9 días y el de Triadimenol 5 días (Figura 7d).

Porcentaje de inóculo viable del oídio. La Eficacia más alta de control del PIV del oídio en campo como preventivo, también fue demostrada por $\mathrm{F}+\mathrm{T}$, con valores del ABCPE y del ABPEE significativamente menores ( $\alpha=p, 01), 88,87 \%$ superior al Triadimenol y $72,77 \%$ superior a $\mathrm{S}+\mathrm{BS}$. La eficacia de S+BS fue 59,16\% superior al Triadimenol (Tabla 4 y Figura 7e). Sin embargo, el periodo de residualidad de $\mathrm{F}+\mathrm{T}$ al estudiar el PIV en campo disminuyó a 8 días, momento en que alcanzó el UED arbitrario de 1\%. El periodo de residualidad de S+BS también disminuyó a 5 días y el periodo de residualidad de Triadimenol disminuyó a 4 días (Figura 7f).

Tabla 4. Prueba de Duncan del ABCPEE del PI, PS y PIV en campo del oídio en hojas de vid Red Globe

\begin{tabular}{cccccccc}
\hline & \multicolumn{2}{c}{ PI } & \multicolumn{2}{c}{ PS } & \multicolumn{2}{c}{ PIV } \\
\cline { 3 - 8 } $\begin{array}{c}\text { Ingrediente } \\
\text { Activo }\end{array}$ & $\begin{array}{c}\text { Dosis P.C. } \\
\text { (L/ha) }\end{array}$ & $\begin{array}{c}\text { ABCPEE } \\
\text { (\%-dia) }\end{array}$ & $\begin{array}{c}\text { \% Eficacia } \\
\text { respecto aT }\end{array}$ & $\begin{array}{c}\text { ABCPEE } \\
\text { (\%-dla) }\end{array}$ & $\begin{array}{c}\text { \% Eficacia } \\
\text { respecto a T }\end{array}$ & $\begin{array}{c}\text { ABCPEE } \\
\text { (\%-dla) })\end{array}$ & $\begin{array}{c}\text { \% Eficacia } \\
\text { respecto a T }\end{array}$ \\
\hline $\begin{array}{c}\text { Fluopyram + } \\
\text { Tebuconazol }\end{array}$ & 0,60 & $8,54 \mathrm{a}$ & 79,59 & $1,74 \mathrm{a}$ & 82,51 & $2,78 \mathrm{a}$ & 88,87 \\
$\begin{array}{c}\text { Spiroxamine } \\
\text { + B. subtilis }\end{array}$ & $0,36+3,00$ & $24,17 \mathrm{~b}$ & 42,26 & $4,53 \mathrm{~b}$ & 54,47 & $10,21 \mathrm{~b}$ & 59,16 \\
Triadimenol & 0,300 & $41,86 \mathrm{c}$ & 0,00 & $9,95 \mathrm{c}$ & 0,00 & $24,98 \mathrm{c}$ & 0,00 \\
\hline
\end{tabular}

Triadimenol

\section{DISCUSIÓN}

Las horas diarias con temperaturas favorables para el oídio durante los 20 días de estudio en campo, superaron ampliamente los tres días consecutivos con $6 \mathrm{~h}$ entre $21-30^{\circ} \mathrm{C}$, necesarios para que se active el índice de Riesgo del Oídio establecido por Gubler et al. (1999), caracterizándose por una alta presión de la enfermedad a inicios de la fase de producción. Bajo estas condiciones, Fluopyram + Tebuconazol ha demostrado su eficacia en el control del oídio de la vid en dos escenarios diferentes. El primero, en el laboratorio como curativo, aplicado al 0,1\% bajo condiciones de alto potencial del inoculo de oídio, con una eficacia de control de la conidias viables superior y con mayor periodo de residualidad (ocho días). 

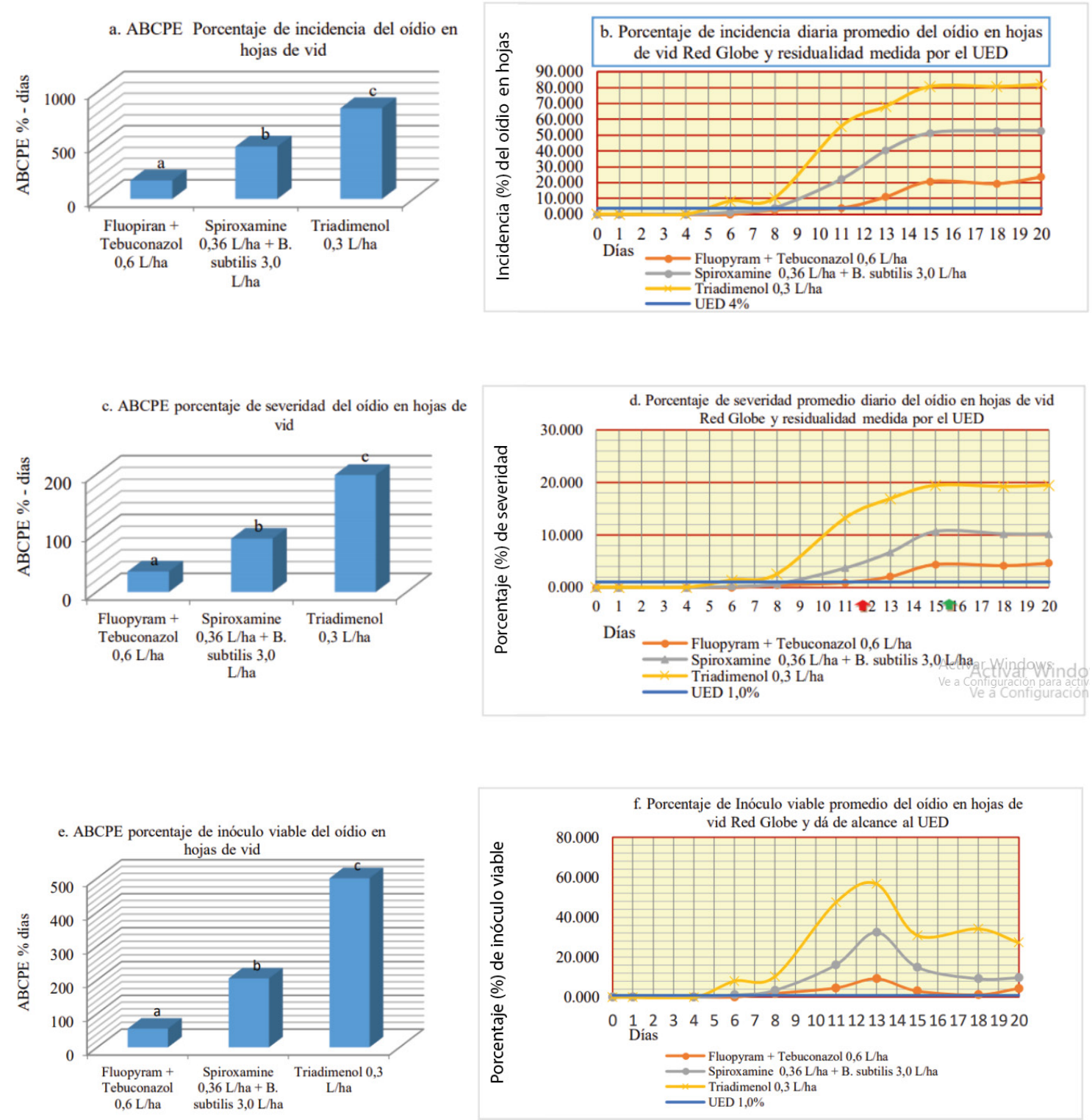

Figura 7. a) alta eficacia de F+T en el control preventivo del PI del oídio en campo demostrada por su menor valor del ABCPE, 75,59\% superior a Triadimenol y 64,66\% superior a $\mathrm{S}+\mathrm{BS} A B C P E$, b) alto periodo de residualidad de $\mathrm{F}+\mathrm{T}$ aplicado como preventivo, de 11 días, medido cuando el PI llegó al UED arbitrario de 4\%, c) alta eficacia de F+T en el control preventivo del PS del oídio en campo demostrada por su menor valor del ABCPE, 82,51\% superior a Triadimenol y 61,59\% superior a $\mathrm{S}+\mathrm{BS}$, d) alto periodo de residualidad de $\mathrm{F}+\mathrm{T}$ aplicado como preventivo, de 11 días, medido cuando el PS llegó al UED arbitrario de 1\%, e) alta eficacia de F+T en el control preventivo del PIV del oídio en campo demostrada por su menor valor del ABCPE, $88,87,59 \%$ superior a Triadimenol y $72,77 \%$ superior a $S+B S, f$ ) el periodo de residualidad de F+T aplicado como preventivo disminuyó a 8 días medido cuando el PIV Ilegó al UED arbitrario de 1\%. La fecha roja indica que a los 12 días se aplicó Spiroxamine en los lotes de plantas tratadas al inicio con Fluopyram + Tebuconazol y con Triadimenol y; se aplicó Metrafenona en plantas al inicio tratadas con Spiroxamine + B. subtilis. La flecha verde indica que a los 16 días se aplicó Tebuconazol + Trifloxystrobin en todo el campo experimental 
El segundo, en campo, a dosis de 0,60 L/ha con incidencia cero y como preventivo, pero con condiciones climatológicas muy favorables para el desarrollo del oídio, su eficacia y residualidad sobre el porcentaje de incidencia del oídio, porcentaje de severidad y porcentaje de inóculo viable sobre los demás fungicidas fue muy marcada. Al comparar los tres métodos de evaluación en la Figura 7, se observa que, después de los 13 días el porcentaje de inóculo viable del oídio tuvo una tendencia general a la baja, pero muy marcada en $\mathrm{F}+\mathrm{T}$, lo que no se observó en el porcentaje de incidencia ni en el porcentaje de severidad. La menor residualidad de F+T encontrada por el PIV del oídio en campo a los 8 días, se debe a que es un método más riguroso, lo que indica que sería el más adecuado para evaluar ensayos comparativos sobre la eficacia de fungicidas en el control del oídio y para decidir el momento ideal de una nueva aplicación, evitando elevar los costos de control y contribuyendo a la economía de la empresa. La única dificultad para la adopción de este método es el requerimiento de personal entrenado y el uso de lupas de 20 aumentos para un mejor estimado del grado de inóculo viable que aparece ya sea producto de infecciones primarias o después de la aplicación de fungicidas. También se destaca la influencia de F+T en la mejora de la eficacia de los fungicidas que se aplican posteriormente, al observar que, las plantas tratadas al inicio con $\mathrm{F}+\mathrm{T}$, a los 12 días con Spiroxamine y a los 16 días con Tebuconazol + Trifloxystrobin, a pesar de haber superado el UED, tuvieron niveles comparativos más bajos que los porcentajes de incidencia, severidad e inóculo viable hasta los 20 días que los demás tratamientos.

La acción de F+T se debe a la acción conjunta del Fluopyram, inhibidor del complejo II de la succinato deshidrogenasa que está presente en la cadena respiratoria mitocondrial de los hongos y, a la acción del Tebuconazol que actúa inhibiendo la demetilasa en la biosíntesis del ergosterol de los hongos (FRAC, 2018). Teniendo en cuenta que, para el cultivo de vid, Tebuconazol tiene un LMR de 2,0 ppm y Fluopyram 1,5 ppm y un periodo de carencia de 7-10 días estudiado para Luna Experience en frijol francés por Katna et al, (2018), F+T puede ser aplicado en dos momentos durante la fase productiva en Piura. El primero, como fungicida preventivo, antes que aparezcan los primeros síntomas del oídio, ofreciendo una protección efectiva de hasta 10-11 días y, el segundo, como curativo en la fase crítica después de la caída de caliptra hasta 8 días. Por otra parte, se resalta la importancia de la labor de deshoje realizada 15 días después de la aplicación de los tratamientos, la misma que ayudó a reducir el inóculo viable en el haz de las hojas que estaban sombreadas de la luz solar por otras hojas ubicadas sobre ellas antes que se ejecute esta labor. El sombreado de la luz solar directa incrementa la enfermedad, en parte como resultado de la protección de la radiación ultravioleta, hacia la cual el oídio es parcialmente vulnerable (Austin, 2010; Willocquet et al., 1996). La exposición a la luz UV reduce la germinación conidial, germinación del apresorio y subsecuente expansión de la colonia y, prolonga el periodo de latencia. Temperaturas de la superficie de la hoja a la luz solar directa pueden ser $10-15^{\circ} \mathrm{C}$ más altas que a la sombra, y puede dar lugar a condiciones letales para el hongo en tejido expuesto frente a tejidos sombreados (Austin, 2010). EL manejo de la canopia es un importante componente en los programas de manejo integrado del oídio de la vid. También, es muy importante la labor de raleo de bayas y despunte de racimos para restarle condiciones al oídio durante la fase de desarrollo de bayas.

\section{CONCLUSIONES}

1. Fluopyram + Tebuconazol al 0,1\% demostró mayor eficacia y un periodo de residualidad de ocho días como fungicida curativo en el control de conidias viables del oídio de la vid variedad Red Globe en laboratorio. 
2. Fluopyram + Tebuconazol aplicado preventivamente en campo a dosis de 0,60 L/ha demostró mayor eficacia protectora y un periodo de residualidad de 11 días del oídio de la vid variedad Red Globe medido por los porcentajes de incidencia y de severidad y, de 8 días medido por el porcentaje de inóculo viable.

3. El mayor periodo de residualidad de Fluopyram + Tebuconazol influye en la mejora de la eficacia de los fungicidas que se aplican posteriormente.

\section{REFERENCIAS BIBLIOGRAFICA}

Abbott W.S. (1925). A method of computing the effectiveness of an insecticide. J. Econ. Entomology 18: 265267.

Austin C.N. 2010. Sunlight's influence on grapevine powdery mildew: direct effects on pathogen development and attendant consequences of canopy management and vineyard variability. PhD Thesis, Cornell University, Ithaca, NY.

Campbell C.L., and L. V. Madden. 1989. Introduction to Plant Disease epidemiology. John Willey \& Sons. 532 pp.

Campbell P., Bendek C., y B.A. Latorre. 2007. Riesgo de oídio (Erysiphe necator) de la vid en relación con el desarrollo de los racimos. Cien. Inv. Agr. 34(1):5-11.

FRAC Code List. 2018: Fungicides sorted by mode of action (including FRAC Code numbering). In: http://www.phi-base.org/images/ fracCodeList.pdf (acceso el 3 de marzo del 2020).

Gadoury D.M., Cadle-Davidson L., Wilcox W.F., Dry I.B., Seem R.C., and M.G. Milgroom. 2012. Grapevine powdery mildew (Erysiphe necator): a fascinating system for the study of the biology, ecology and epidemiology of an obligate biotroph. Molecular Plant Pathology 13(1): 1-16.
Gubler W.D., Rademacher, M.R. and S.J. Vasquez. 1999. Control of Powdery Mildew Using the UC Davis Powdery Mildew Risk Index. APSnet Features. In: https://www.apsnet.org/ edcenter/apsnetfeatures/Pages/UCDavisRisk. aspx (acceso el 11 de febrero del 2020).

Pearson R.C., and W. Gärtel. 1985. Occurrence of hyphae of Uncinula necator in buds of grapevine. Plant Disease 69: 149-151.

Katna S., Kumar Dubey J., Kumar Patyal1 S., Devi1 N., Chauhan A., A. Sharma. 2018. Environmental Science and Pollution Research (2018) 25:27594-27605.

Kunova A., Pizzatti C., Bonaldi M., and P. Cortesi. 2015. Metrafenone Resistance in a Population of Erysiphe Necator in Northern Italy. Pet Manag Sci, 72 (2), 398-404

Townsend G.R, and J.W. Heuberger. 1943. Methods for estimating losses caused by diseases in fungicidal treatments. Plant Disease Rept. 27:340-343.

Willocquet L., Colombet D., Rougier M., Fargues J. and M. Clerjeau. 1996. Effect of radiation, especially UV-B, on spore germination and mycelial growth of the grape powdery mildew. Eur. J. Plant Pathol. 102, 441-449.

\section{CORRESPONDENCIA:}

Dr. Javier Javier-Alva jjavieralva@hotmail.com 